\title{
Multiresidue antibiotic-metabolite quantification method using ultra-performance liquid chromatography coupled with tandem mass spectrometry for environmental and public exposure estimation
}

\author{
Elizabeth Holton ${ }^{1}$ (D) Barbara Kasprzyk-Hordern ${ }^{1}$ (D)
}

Received: 14 April 2021 / Revised: 24 June 2021 / Accepted: 21 July 2021 / Published online: 8 September 2021

(C) The Author(s) 2021

\begin{abstract}
This manuscript describes a new multiresidue method utilising ultra-performance liquid chromatography tandem mass spectrometry (UPLC-MS/MS) via multiple reaction monitoring (MRM), for the identification and quantification of 58 antibiotics and their 26 metabolites, in various solid and liquid environmental matrices. The method was designed with a 'one health' approach in mind requiring multidisciplinary and multisectoral collaborative efforts. It enables comprehensive evaluation of antibiotic usage in surveyed communities via wastewater-based epidemiology, as well as allowing for the assessment of potential environmental impacts. The instrumental performance was very good, demonstrating linearity up to $3000 \mu \mathrm{g} \mathrm{L}^{-1}$, and high accuracy and precision. The method accuracy in several compounds was significantly improved by dividing calibration curves into separate ranges. This was accompanied by applying a weighting factor $(1 / x)$. Microwave-assisted and/or solid-phase extraction of analytes from liquid and solid matrices provided good recoveries for most compounds, with only a few analytes underperforming. Method quantification limits were determined as low as $0.017 \mathrm{ng} \mathrm{L}^{-1}$ in river water, $0.044 \mathrm{ng} \mathrm{L}^{-1}$ in wastewater, $0.008 \mathrm{ng} \mathrm{g}^{-1}$ in river sediment, and $0.009 \mathrm{ng} \mathrm{g}^{-1}$ in suspended solids. Overall, the method was successfully validated for the quantification of 64 analytes extracted from aqueous samples, and 45 from solids. The analytes that underperformed are considered on a semi-quantitative basis, including aminoglycosides and carbapenems. The method was applied to both solid and liquid environmental matrices, whereby several antibiotics and their metabolites were quantified. The most notable antibioticmetabolite pairs are three sulfonamides and their $\mathrm{N}$-acetyl metabolites; four macrolides/lincomycins and their $\mathrm{N}$-desmethyl metabolites; and five quinolone metabolites.
\end{abstract}

Keywords Antibiotic $\cdot$ Metabolite $\cdot$ Liquid chromatography $\cdot$ Mass spectrometry $\cdot$ Wastewater-based epidemiology $\cdot$ Water fingerprinting

\section{Introduction}

Antimicrobial resistance (AMR) is a major global topic, concerning the increasing pathogenic tolerance to antibiotics. Excessive and inappropriate antibiotic use heightens the emergence of antimicrobial resistance genes in pathogenic organisms, resulting in reduced drug susceptibility. Water

Barbara Kasprzyk-Hordern

b.kasprzyk-hordern@bath.ac.uk

1 Department of Chemistry, University of Bath, Bath BA2 7AY, UK fingerprinting or wastewater-based epidemiology (WBE) techniques offer an innovative approach for tracking markers of disease and AMR, such as antimicrobials, pollutants, pathogenic DNA, and resistance genes. WBE is a relatively new field of research. It has been used to estimate illicit drug and lifestyle chemical usage trends [1-4], community-wide exposure to pesticides $[5,6]$, industrial chemicals (e.g. BPA or perfluorinated chemicals) [7-9], or mycotoxins [10]. Moreover, with the advent of the SARS-CoV-2 coronavirus pandemic at the end of 2019 (COVID-19), WBE has been applied globally to track community infection with SARCoV-2 $[11,12]$. However, there is very limited published research on antibiotics and AMR $[13,14]$. WBE has a significant potential to determine the spatiotemporal distribution 
patterns of antibiotics and resistance genes, such as via predictive modelling of early warning systems for infectious disease, as well as identifying hotspots of AMR emergence and dissemination. As such, WBE should become one of the key tools utilised in the 'one health' approach. For example, through longitudinal monitoring, correlations could be determined between exposure, environmental pressures, and the occurrence of resistance genes. Such rich, datadriven research can help to provide mitigation measures spanning from 'at source' solutions (e.g. reduction of antibiotic usage and educational campaigns aimed at limitation of direct disposal of unused antibiotics) through technology-based 'end-of-pipe' treatment and environmental health-driven measures.

However, there are several research gaps that require attention before the tool can be implemented on a wider regional, national, or indeed international scale. For example, the usage, excretion, and fate of antibiotics are an important aspect of AMR dissemination. In regions where prescription data and pharmacy records are not collated or publicly available, estimations of drug usage can be back-calculated from the quantities in wastewater. Resistance-causing genetic mutations occur naturally, but also as a result of environmental factors, such as selective pressures [15]. There are many social and clinical factors that induce selective pressures for AMR emergence. Incorrect, excessive, and over-use of antimicrobials can harbour inherent resistance, including sub-therapeutic doses (e.g. livestock feed supplements); insufficient prevention and control of infection/disease; poor surveillance facilities in hospitals for diagnoses of resistant strains; ineffective regulatory barriers (e.g. self-prescribing, over-the-counter drugs); inappropriate prescribing (due to access/expense, or prescription without diagnosis); extensive agricultural use; limited availability/uncompleted antibiotic courses; incomplete removal from treated and untreated waste; and stunted development of new pharmaceuticals [15]. But equally, consistent accumulation of excreted or discarded antimicrobials into urban water will create selective pressures. These relationships are documented, particularly in association with wastewater treatment plants (WWTP) [16]. There have also been correlations drawn between the prevalence of polluting agents, such as of biocides and heavy metals, and the spread of AMR [17].

In order to fully embrace the 'one health' approach with WBE tools, new multiresidue methods are required that include both antibiotics and their metabolites, in various solid and liquid matrices. Several methods are already published in this field, but many of them either focus on a low number of analytes; analysis of one matrix; or do not include drug metabolites [18-26]. In this paper, a new analytical method has been developed to monitor a wide spectrum of antibiotics and their metabolites, through quantification in both solid and liquid environmental matrices. Assessments of both public and environmental health can be achieved by monitoring urban contamination and community-wide drug usage. Solid components of water systems (suspended particulate matter and river sediments) are important to include due to analyte partitioning and chemical accumulation. Additionally, by including drug metabolites in analyses, a distinction can be made between drug excretion and drug disposal [8]. This method encompasses analysis of multiple matrices, for parent drugs and several associated metabolites in order to provide a comprehensive WBE tool—supporting 'one health' research and tackling the AMR challenge.

\section{Materials and methods}

\section{Analyte selection}

There were several considerations when selecting targets: metabolism and excretion mechanisms; availability and usage; aquatic toxicity; and drug resistance. It was important to target accessible drugs, from a wide range of chemical classes, and include both broad- and narrow-spectrum antibiotics. A comprehensive list was compiled from several antibiotic groups. Overall, a total of 58 drugs, 26 metabolites, and 21 stable isotope-labelled internal standards were selected (excluding separate forms of drug complexes) (Table S1, see Supplementary Information, ESM).

Ketoconazole (an antifungal) and its metabolite were included with the antibiotics, as it has the same considerations and emergence of resistant organisms. Sulfasalazine, an antiinflammatory, was included alongside other sulfonamides. Tuberculosis (TB) drugs were added due to the significance and high prevalence in several countries, particularly those with limited health resources. Antiretrovirals, emtricitabine, and lamivudine were included because of the significant association of tuberculosis and HIV coinfection [27]. Thalidomide, an immunomodulatory anti-leprosy drug, has been referenced as an adjunctive treatment in some tuberculosis cases [28]. Major metabolites were selected where possible. Enrofloxacin (primarily a veterinary antibiotic) was included due to its metabolism into ciprofloxacin. DCycloserine, a TB antibiotic and the active metabolite of prodrug terizidone, was retained in the method after terizidone was excluded (and so is unpaired in antibiotic-metabolite analyses).

\section{Materials}

Analytical standards and deuterated (stable isotope-labelled) standards were purchased from Sigma-Aldrich (Gillingham, UK), TRC (Toronto, Canada), LGC (Middlesex, UK), or MCE (Cambridge, UK); the list of which is collated in ESM Table S1. Methanol, MeOH, was HPLC-grade 
(Sigma-Aldrich). Water, $\mathrm{H}_{2} \mathrm{O}$, was of $18.2 \mathrm{M} \Omega$ quality (Elga, Marlow, UK). Glassware was deactivated using $5 \%$ dimethylchlorosilane (DMCS) in toluene (SigmaAldrich) to mitigate the loss of basic chemicals onto $-\mathrm{OH}$ sites present on glass surfaces. This consisted of rinsing once with DMCS, twice with toluene and three times with $\mathrm{MeOH}$. Several mobile-phase buffers were tested during method development, including ammonium acetate $\left(\mathrm{NH}_{4} \mathrm{OAc}\right)$, ammonium fluoride $\left(\mathrm{NH}_{4} \mathrm{~F}\right)$, formic acid $(>95 \%, \mathrm{HCOOH})$, and acetic acid $(1.0 \mathrm{M}$, $\left.\mathrm{CH}_{3} \mathrm{COOH}\right)$ purchased from Sigma-Aldrich. Oasis HLB $(60 \mathrm{mg}, 3 \mathrm{~mL})$ SPE cartridges, polypropylene LC vials, and Whatman GF/F $0.7-\mu \mathrm{m}$ filters were purchased from Waters (Manchester, UK).

\section{Sample collection and extraction methods}

Both solid and aqueous samples were analysed (Fig. 1). Compound partitioning between solid and liquid phases is based on their specific physical-chemical properties. Therefore, extraction from various matrices is desirable for full quantification of analytes within a whole system. However, aqueous samples were spiked with internal standards on the day of collection, at least $30 \mathrm{~min}$ before the filtration step. This accounted for the proportion of analyte partitioning into the solid phase and being lost during filtration. Consequently, this design can more accurately represent the total quantity of analyte present, independent of the corresponding solids analyses. This method therefore allows for the quantification of the total mass of antibiotics via the analysis of the aqueous phase and suspended particulate matter (SPM) fraction via the analysis of SPM.

Samples were collected via either 24-h time-proportional composite sampling (wastewater and associated solids) or grab sampling (river water and associated solids). Grab sampling is indicative of the system at a single time point. A composite sample is obtained by combining discrete grab samples collected at regular 15-min time intervals (using an automated sampler), which is representative of a system over time. Wastewater influent and effluent were collected from a WWTP in South West England via composite sampling. Influent SPM was extracted from a grab sample (3.3 g from $12 \mathrm{~L}$, homogenised). SPM would typically be extracted from composite samples. However, due to the large quantities required for validation studies, a grab sample was used in this case. River water and sediment samples were also collected in South West England. Aqueous samples were collected via composite sampling and sediments from a singular grab sample (4.7 dry grammes, homogenised).

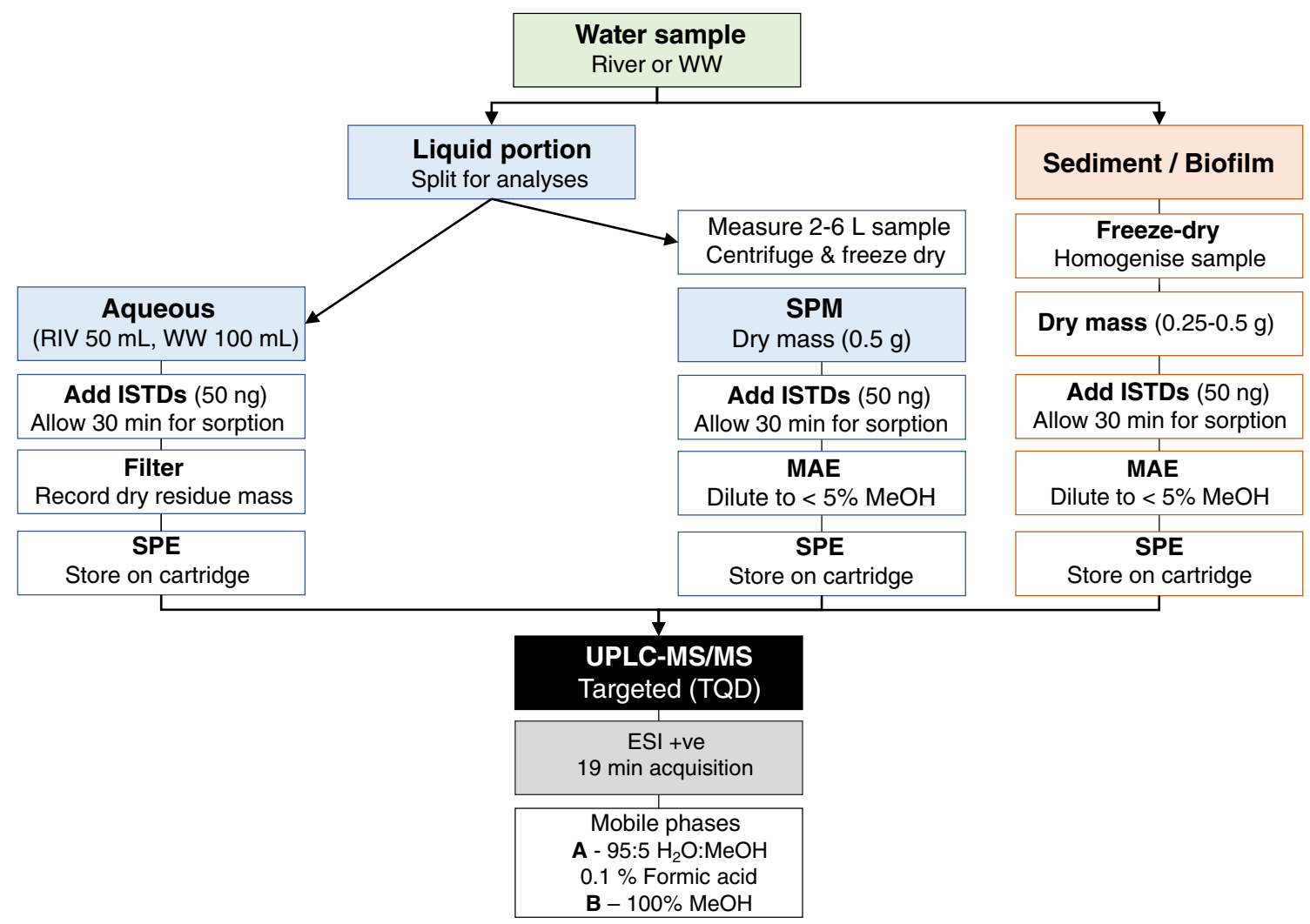

Fig. 1 Schematic for matrix-specific sample processing. WW, wastewater; RIV, river; SPM, suspended particulate matter; ISTDs, stable isotope internal standards; MAE, microwave-assisted extraction; SPE, solid-phase extraction; TQD, triple quadrupole detector 


\section{Extraction procedure for liquid matrices (analyte concentration via SPE)}

Wastewater samples $(50 \mathrm{~mL})$ and river samples $(100 \mathrm{~mL})$ were spiked with $50 \mathrm{ng}$ of each internal standard $(50 \mu \mathrm{L}$ of $1 \mu \mathrm{g} \mathrm{mL}{ }^{-1}$ mix), shaken, and left to partition for at least $30 \mathrm{~min}$ at $4{ }^{\circ} \mathrm{C}$. Samples were then filtered using an ovendried, pre-weighed filter paper (Whatman GF/F $0.7 \mu \mathrm{m}$ ). After drying, filter papers were re-weighed, recording the mass of the suspended particulate matter removed. The filtrates were loaded under vacuum onto conditioned Oasis HLB cartridges $\left(2 \mathrm{~mL} \mathrm{MeOH}\right.$, followed by $2 \mathrm{~mL} \mathrm{H}_{2} \mathrm{O} 0.1 \%$ formic acid) at approx. $5 \mathrm{~mL} \mathrm{~min}{ }^{-1}$. Sampling bottles and apparatus were washed with $\mathrm{H}_{2} \mathrm{O}(<10 \mathrm{~mL}$ total $)$ during transfers. Cartridges were dried under vacuum, sealed with parafilm, and stored at $-20{ }^{\circ} \mathrm{C}$ until elution or shipment. Elution was instigated using $4 \mathrm{~mL} \mathrm{MeOH}$, where the eluate was collected in deactivated glass vials. Extracted samples were dried under nitrogen, at $40{ }^{\circ} \mathrm{C}$ and re-suspended (500 $\mu \mathrm{L}$ 80:20 $\mathrm{H}_{2} \mathrm{O}: \mathrm{MeOH}$ ) into $\mathrm{LC}$ vials (Waters).

\section{Extraction procedure for solid matrices}

Two methods were tested for analyte extraction from solid matrices: ultra-sonication-assisted extraction (UAE) and microwave-assisted extraction (MAE). Due to variations in solvent workup, the two methods showed large differences in the chromatography (ESM Figure S4). The MAE method was chosen for analyses due to greater chromatographic peak symmetry, lower method quantification limits, and better analyte recovery.

Suspended particulate matter (SPM) was collected by centrifugation of $1-6 \mathrm{~L}$ of aqueous sample $\left(35,000 \mathrm{~m} / \mathrm{s}^{2}, 15 \mathrm{~min}\right)$ and accumulating a pellet. Other solids (river sediment and biofilm) were grab samples, independent of volume. Collected solids were dried under vacuum, freeze-dried, homogenised, split for replicate analysis $(0.25-0.50 \mathrm{~g})$, and spiked with $50 \mathrm{ng}$ of each internal standards $(50 \mu \mathrm{L}$ of $1 \mu \mathrm{g}$ $\mathrm{mL}^{-1}$ mix). Samples were stored at $-20{ }^{\circ} \mathrm{C}$ until extraction or shipment. The microwave-assisted extraction method used was modified from a method developed by Petrie et al. [29] by a substitution for HLB cartridges. The technique utilised an $800 \mathrm{~W}$ MARS 6 microwave (CEM, UK), which processed acidified samples over a temperature gradient to $80{ }^{\circ} \mathrm{C}$. Samples were vortexed, filtered $(0.7 \mu \mathrm{m})$, and diluted with $\mathrm{H}_{2} \mathrm{O}(0.1 \%$ formic $)$ to $<5 \% \mathrm{MeOH}$. Solid-phase extraction was then performed using the same method as for aqueous filtrates.

\section{Liquid chromatography-mass spectrometry method}

Liquid chromatography-mass spectrometry (LC-MS/MS) was performed using a Waters, ACQUITY UPLC ${ }^{\text {TM }}$ system coupled to a Xevo TQD-ESI Mass Spectrometer and using a reverse-phase BEH C18 column $(50 \times 2.1 \mathrm{~mm}, 1.7 \mu \mathrm{m})$ with Acquity column in-line $0.2-\mu \mathrm{m}$ pre-filter (Waters, Manchester, UK). Conditions were optimised for fast chromatographic separation and high sensitivity across a range of drug classes.

The coordinating programme used was MassLynx V4.1, and the data processing programmes were QuanLynx and TargetLynx (Waters Lab Informatics, UK). The integration parameters in QuanLynx (smoothing, apex track, and window extent) were optimised per analyte to minimise the effects of analyst subjectivity during data processing.

\section{Liquid chromatography}

The chromatography was optimised in terms of mobile-phase composition, needle washes, sample composition, flow rate, and inlet gradient (ESM, Table S3 and supplementary text).

The best separation and organic purge was achieved using 95:5 $\mathrm{H}_{2} \mathrm{O}: \mathrm{MeOH}$ with $0.1 \%$ formic acid (mobile phase A) and $100 \% \mathrm{MeOH}$ (mobile phase B). Strong and weak needle washes were selected to compliment the system. The strong wash used was 1:1:1:1 MeOH:ACN:IPA: $\mathrm{H}_{2} \mathrm{O}+0.1 \%$ formic acid, and the weak wash was prepared to mimic the starting mobile phase $\left(95: 5 \mathrm{H}_{2} \mathrm{O}: \mathrm{MeOH}\right)$. The injection volume was $20 \mu \mathrm{L}$, via a partial loop injection. Sample composition performed best at 60:40 but was prepared at 80:20 $\mathrm{H}_{2} \mathrm{O}: \mathrm{MeOH}$ to enable samples to be co-analysed with pre-established methods. The best results, in terms of system pressure, peak separation, and peak shape, were determined at $0.2 \mathrm{~mL} \mathrm{~min}^{-1}$.

Numerous elution profiles were tested to maximise overall peak separation whilst retaining Gaussian shape. Starting conditions were $0 \% \mathrm{~B}$, held for $1 \mathrm{~min}$, followed by an 8.5 -min gradient to $40 \% \mathrm{~B}$; 3.5-min gradient to $100 \% \mathrm{~B}$; 3-min hold; and finally returning to $0 \% \mathrm{~B}(0.5 \mathrm{~min})$ to re-equilibrate for $2.5 \mathrm{~min}$. The total run time was $19 \mathrm{~min}$. Fig. 2 shows the distribution of peaks relative to the inlet gradient and internal standard retention times (top), as well as the example chromatographs, organised by drug class (bottom).

\section{Mass spectrometry}

Triple quadrupole detector (TQD) mass spectrometry offered fully targeted analyses. The source desolvation temperature was $400{ }^{\circ} \mathrm{C}$. The cone gas flow was $100 \mathrm{~L} \mathrm{~h}^{-1}$ and desolvation gas flow was $1000 \mathrm{~L} \mathrm{~h}^{-1}$. Nitrogen was used as the nebulising and desolvation gas, and argon as the collision gas. Specific operating conditions, such as MRM masses and collision energies, were analyte-specific (ESM Table S6).

The parameters were optimised for both general and compound-specific conditions, which included ionisation mode; multiple reaction monitoring (MRM) targets and 

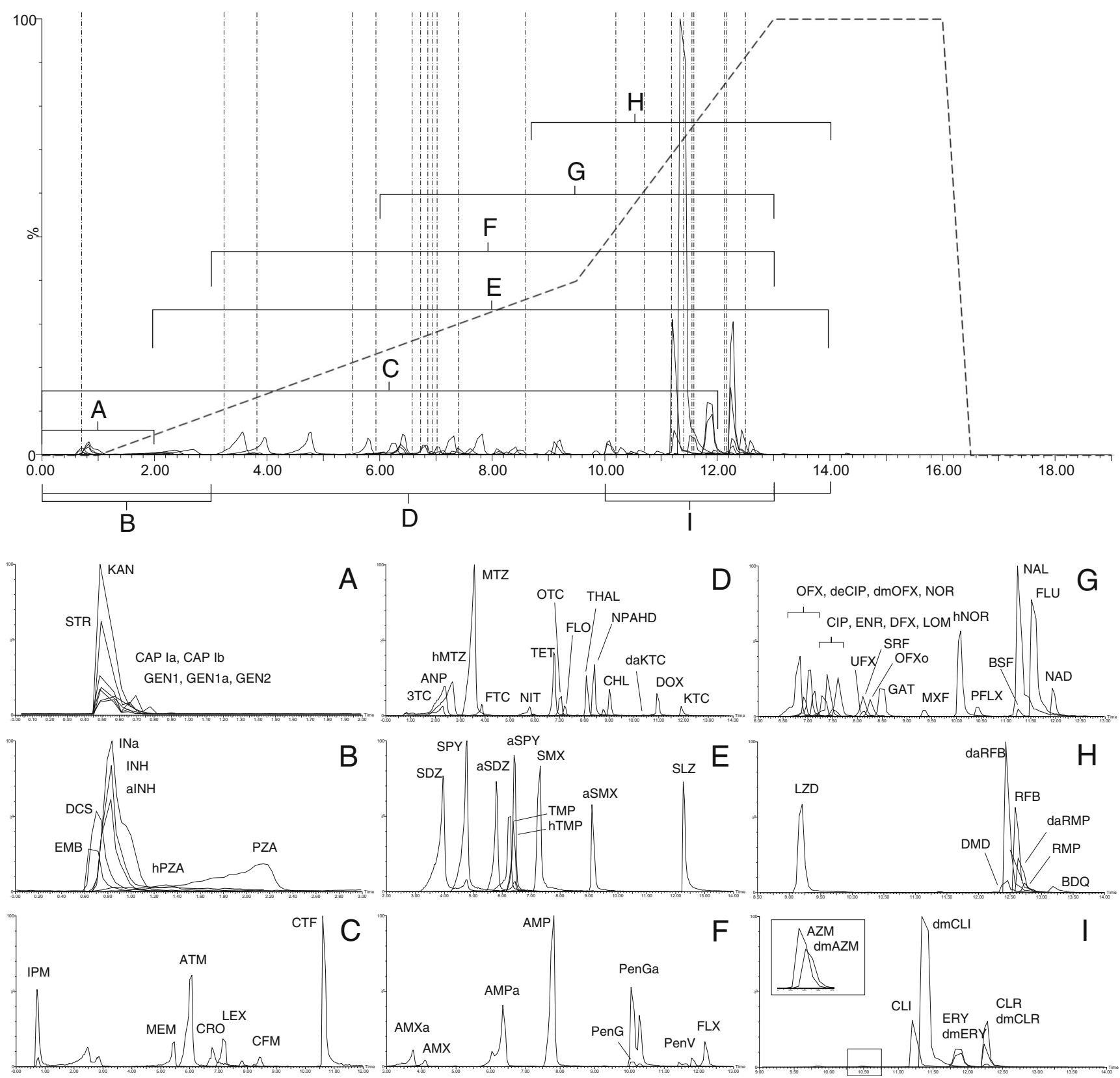

Fig. 2 Chromatographic representation of a mobile-phase standard, scaled by relative intensity. Top: overlaid extracted ion chromatograms (EICs) of the full range (0-19 min); highlighting regions by chemical class (A-I); the distribution of internal standards (vertical dashed lines), as well as the elution gradient (dotted line), which displays mobile-phase composition (\% B) against time (min). Bottom: overlaid chromatograms, sorted by chemical class. Aminoglycosides (A); TB drugs early-eluting (B); cephalosporins and carbapenems (C); ARVs, azoles, nitrofurans, cyclines, and amphenicols (D); sulfonamides (E); penicillins (F), quinolones $(\mathrm{G})$; TB drugs late-eluting $(\mathrm{H})$; macrolides and lincomycins (I). The EICs have not been smoothed, contrary to the method.

Each analyte was tuned for the two most abundant MRM transitions (precursor to product ion $\mathrm{m} / \mathrm{z}$ ), and their associated cone voltage and collision energies (ESM Table S6). Capillary voltage was assigned at $3.8 \mathrm{kV}$. Due to high density of MRM channels (116 channels within $19 \mathrm{~min})$, the retention time windows were staggered $( \pm 0.1 \mathrm{~min})$ where possible to reduce dramatic changes in the number of active channels/dwell time. Analyte acquisition windows were broadened to account for 
matrix shift. Acquisition parameters, such as peak width and points-per-peak, were tested between 20-60 s and 20-30 points, respectively. They were set at $60 \mathrm{~s}$ and $20 \mathrm{ppp}$ to achieve the most Gaussian peak shapes.

In order to calculate any parent/metabolite impurities in the purchased standards, calibration curves were conducted separately and assessed for the presence of metabolites in parent stocks (and vice versa).

\section{Data processing}

Mean peak smoothing parameters (iterations and width) were minimised to preserve resolution, whilst sufficiently removing noise. Data integration was automated (other than removal of false positives) using QuanLynx, to maximise the objectivity of the process. Validation parameters were determined per analyte to aid peak identification, including signal to noise ( $\geq 3$ for detection, $\geq 10$ for quantification); compoundspecific sensitivity limits (IDL and IQL); and to be within stated thresholds for relative retention time and target ion ratio. All data points are accompanied by a quantitative pass/fail assessment for each parameter.

\section{Instrument and method performance}

The following instrument performance parameters were tested: linearity and range, intra- and inter-day accuracy and precision, IDLs, and IQLs. Method performance was tested using method sensitivity (MDLs and MQLs) and method recovery. Formulae used to assess instrument and method performance are gathered in ESM Table S7.

Linearity and instrumental sensitivity were determined for each analyte by replicate injections of calibration standards over separate days; performed between 0 and $1000 \mathrm{ng} \mathrm{mL}^{-1}$ $(n=18 * 3)$ and extended through $500-3000 \mathrm{ng} \mathrm{mL}^{-1}(n=10$ * 3) for selected compounds. Inter- and intra-day precision and accuracy was determined using quality controls at three concentrations $\left(10,50\right.$, and $\left.200 \mathrm{ng} \mathrm{mL}^{-1}\right)$, in replicate (Tables 1, 2, and 3).

Accuracy was determined from physical duplicates of quality controls, injected in replicate, calculated by the measuredconcentration over the theoretical-concentration, as a percentage. Precision was defined as the standard deviation from these same samples. Both inter- and intra-day tests were performed (Table 3).

Matrix recoveries were quantified by spiking aqueous and solid matrices with four quantities of standard $(0,5,25,100 \mathrm{ng})$ at the beginning of extraction processes. For aqueous matrix, samples were spiked before filtration; for solids, samples were spiked before microwave extraction. Method performance was determined by subtracting the average unspiked matrix blanks from the analyte mass and calculating the percentage mass that was recovered throughout sample preparation. Method detection limits were then calculated per matrix using instrumental limits, matrix-specific recovery, and the sample SPE concentration factor (ESM Table S7). Concentration factors per matrix were calculated as the sample volume, or mass, over elution volume, i.e. Milli-Q and river water, 200; wastewater, 100; influent SPM, 0.52; river sediment, 0.54 .

\section{Results and discussion}

\section{Instrument and method performance}

\section{Instrument performance}

Two techniques were used to improve accuracy, particularly at the lower end of instrumental detection: calibration curves were weighted at $1 / x$, and several curves were divided into separate concentration ranges. The weighting scheme was chosen by observing the accuracy of different weighting factors (no weighting, $1 / x$, and $1 / x^{2}$ ) across the range - an example of which is displayed in ESM Figure S1. The unweighted curves typically produced the highest $r^{2}$ values but had large percentage errors at low concentrations. By applying a weighting factor of $1 / x^{2}$, percentage error was more consistent across the entire range, but the error was often above the accepted $\leq \pm 15 \%$ error [30] and would give poor $r^{2}$ values $(<0.95)$. Consequently, a factor of $1 / x$ produced the best accuracy at the lowest variance.

The division of calibration curves was also performed to improve overall accuracy. Calculations of linear regression are dictated by absolute variance; therefore, the slope is generally dominated by data from the highest concentrations. As the instrumental response approaches saturation, the curve can become biased, decreasing the gradient of the line. Across a large concentration range, this effect has negligible impact on the $r^{2}$, but may shift the intercept, and therefore reduce the accuracy around the IQL. An example of this process is shown across three ranges, in ESM Figure S2. The close-up of the origin shows significantly greater accuracy in the lowest calibration range compared to the higher.

Table 3 displays the results for sensitivity (instrument detection limits, IDL, and quantification limits, IQL) as well as performance (accuracy and precision). IQLs were determined from the lowest calibration standard which consistently gave signal-to-noise ratios of $\geq 10$, and IDLs were then calculated for an extrapolated signal-to-noise equal to 3 . IDLs varied from 0.0015 to $12.0 \mu \mathrm{g} \mathrm{L}^{-1}$ and the quantitative ranges extended to between 95 and $3000 \mu \mathrm{g} \mathrm{L} \mathrm{L}^{-1}$ utilising between one and three linear calibration ranges. Generally, macrolides achieved the lowest limits of detection and aminoglycosides the highest. The analytes with the poorest sensitivity (AMGs) elute in the first minute, within the dead volume region. The method was not 


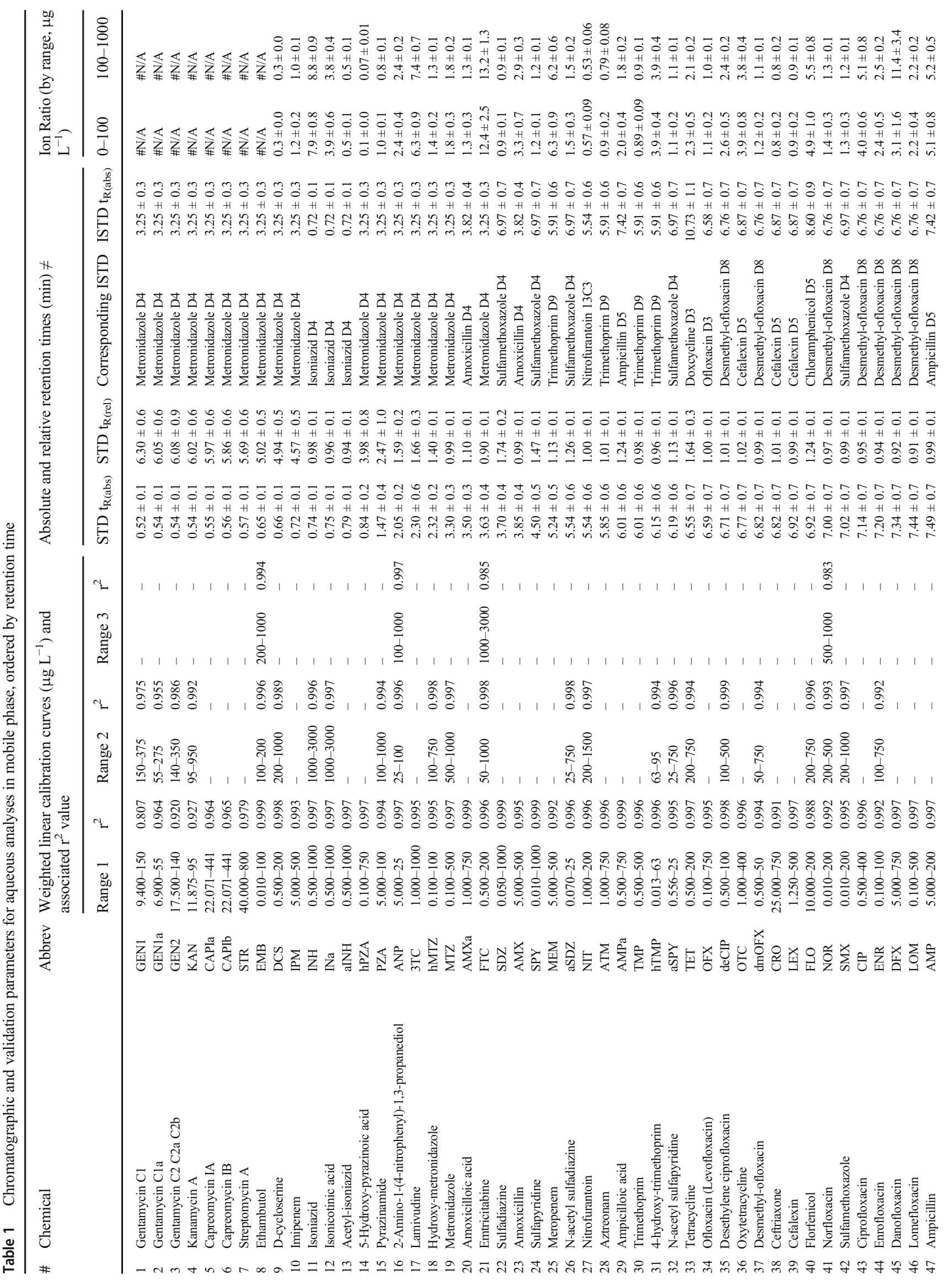




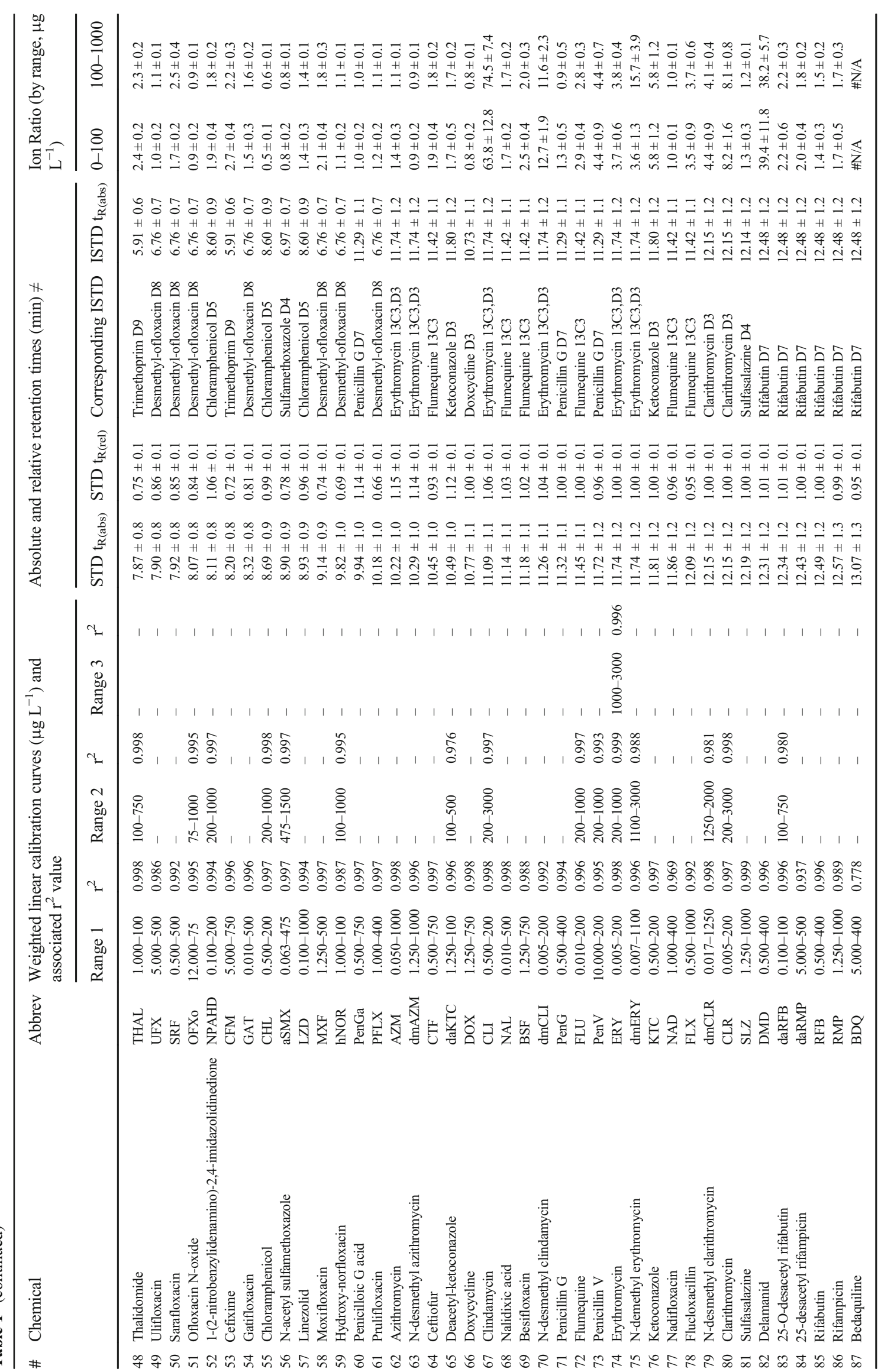




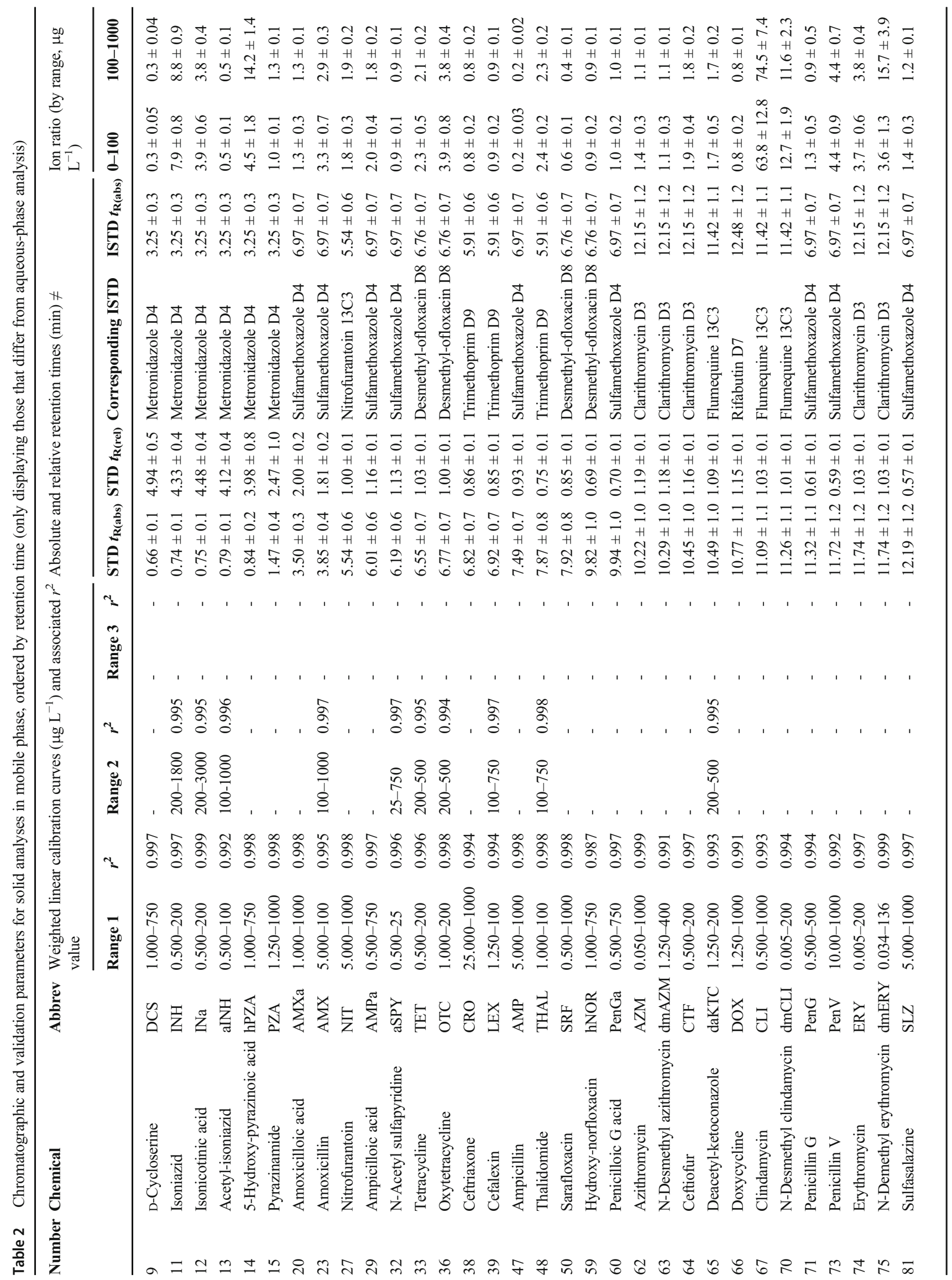




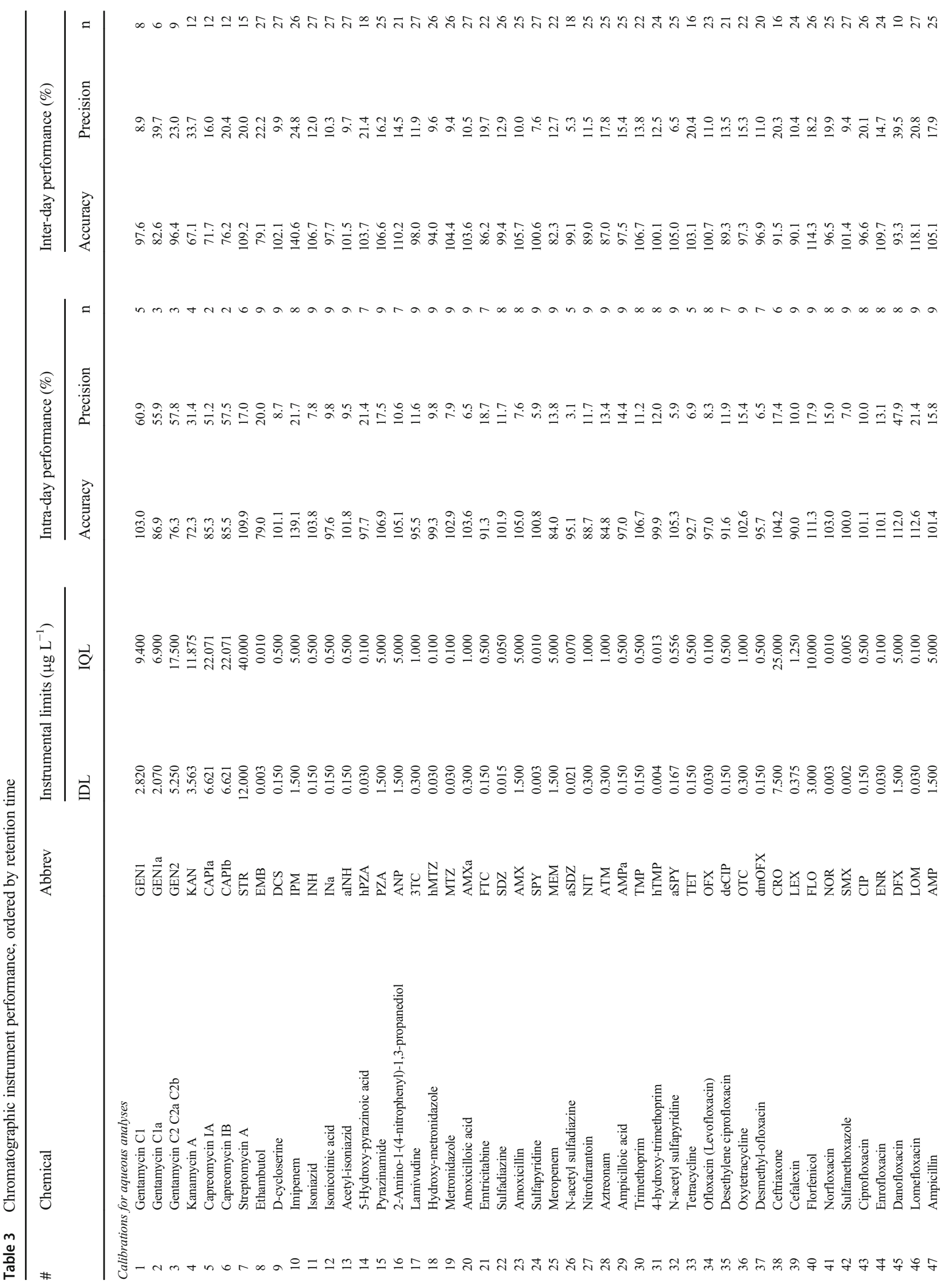




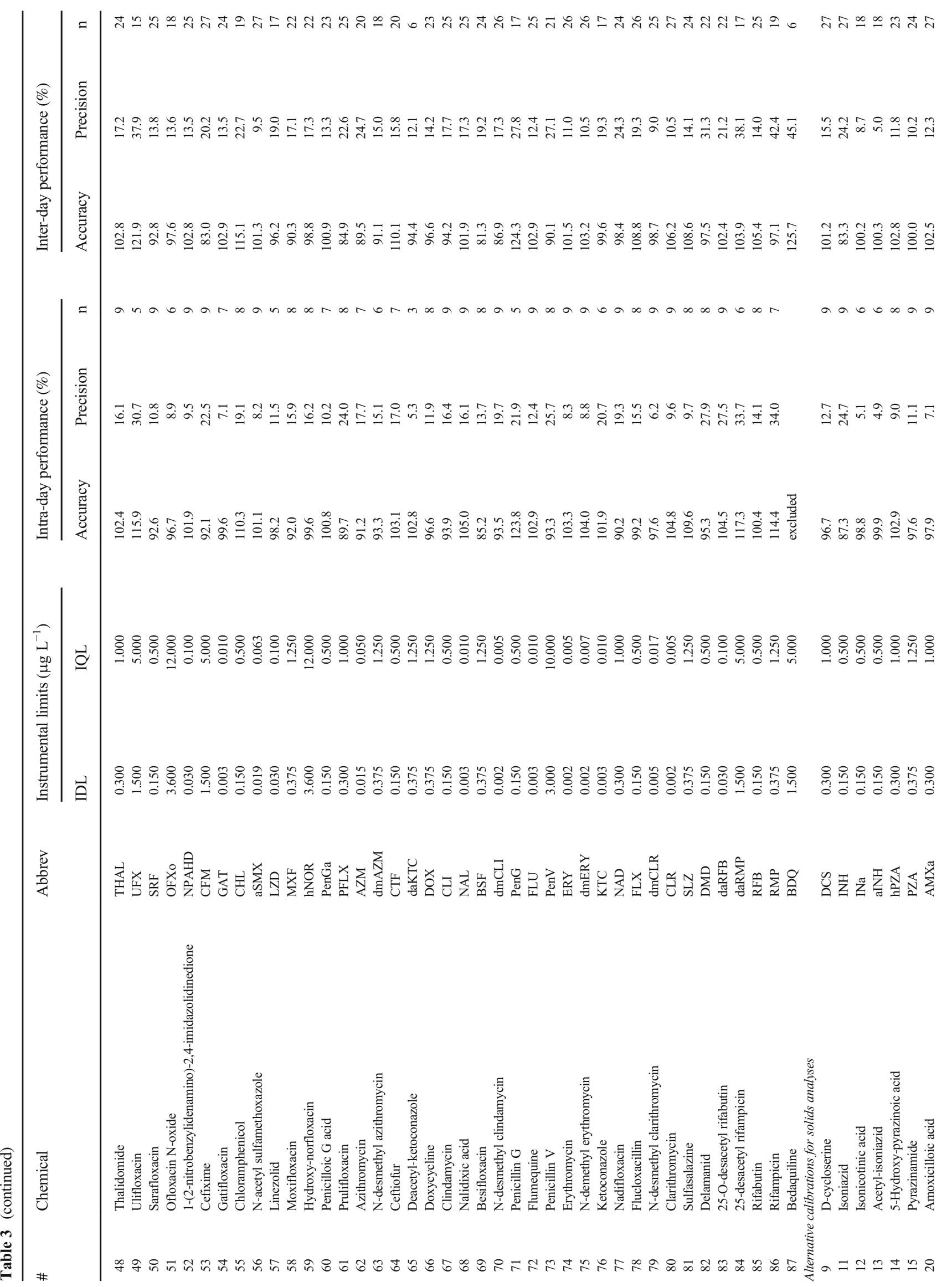




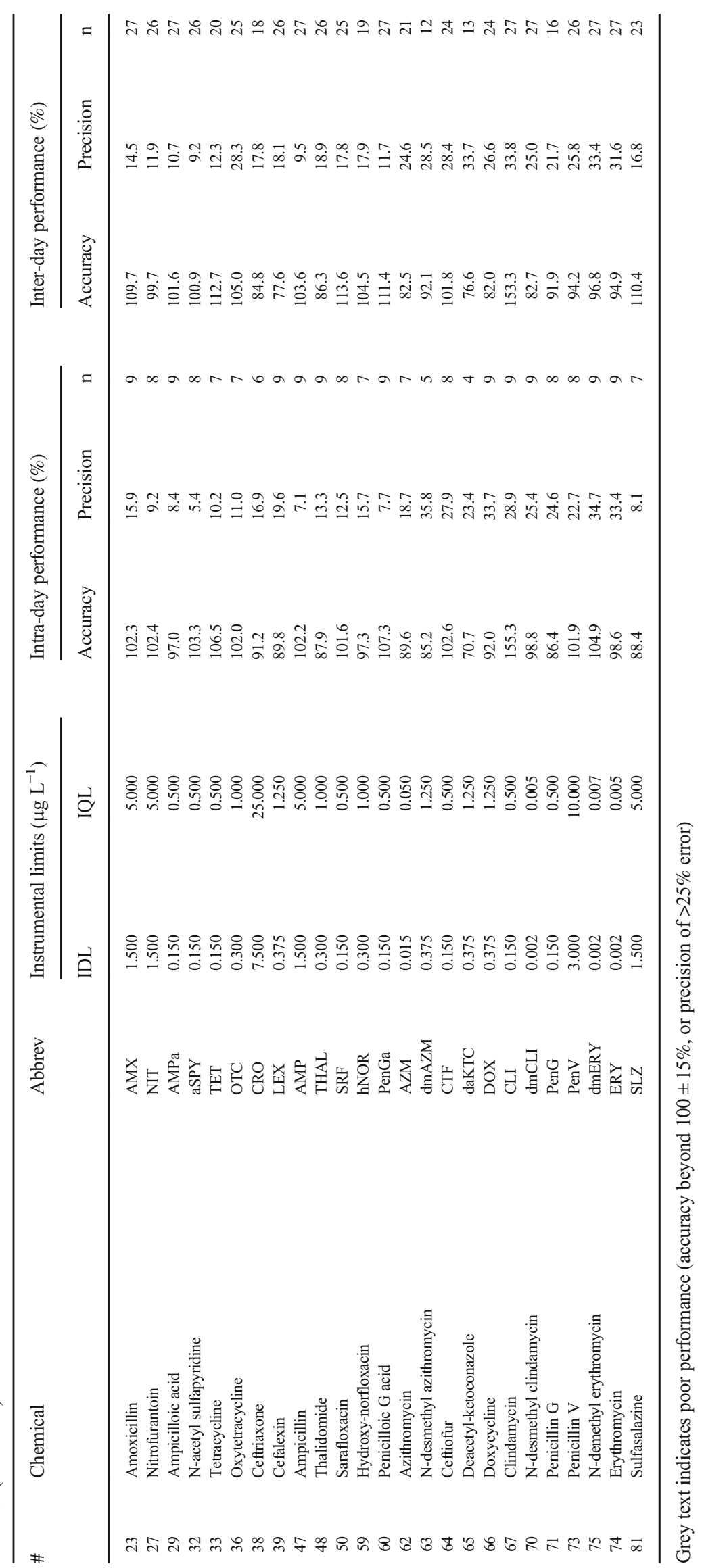


optimised to accommodate these compounds, but they were kept for the intention of semi-quantitative analyses. Other drugs degraded more quickly in the sample composition $\left(80: 20 \mathrm{H}_{2} \mathrm{O}: \mathrm{MeOH}\right)$, notably the isoniazids and $\beta$ lactams. It was therefore important to store standards in methanol, where possible, and remake quality control mixtures before each use.

Inter- and intra-day accuracy and precision were calculated per analyte. Most performed well, 65 and 63 analytes achieved $90-110 \%$ accuracy for intra-day and inter-day studies, respectively. However, several analytes had poor concordance between samples. Approximately $50 \%$ of analytes achieved standard deviations of $<15 \%$ across both studies. This is likely due to the number of active MRM channels per cycle (a total of 120 channels within the 19-min method). To accommodate, channel windows were minimised to maximise dwell time and staggered to reduce noise. Analytes that performed worst ( $\geq 25 \%$ stdev) are formatted in grey text and may be considered semi-quantitative in the corresponding analysis phase. These included aminoglycosides (GEN, KAN, and CAP), $\beta$-lactams (IPM, MEM, ATM, CFM, PenG, PenV), and some TB drugs (EMB, DMD, and rifamycins). Alternate calibrations used for solids were designed to improve analyte quantitation from different matrices. Most examples have comparable instrumental performance, but some analytes were labelled as semi-quantitative during solid analysis (OTC, dmAZM, CTF, daKTC, DOX, CLI, dmCLI, dmERY, and ERY).

\section{Method performance}

Matrix retention times $\left(t_{\mathrm{R}}\right)$ and ion ratios were obtained from spiked matrices. Only data with a signal-to-noise ratio $\geq 10$ were used to calculate these parameters. Accepted tolerances for retention times were typically $\pm 10 \%$. Ion ratios often varied with concentration due to differences in product ion sensitivity or channel-specific interference. Consequently, ion ratio parameters were conditional on sample concentration (stated for regions 0 $100 \mathrm{ng} \mathrm{L}^{-1}$ and 100-1000 $\mathrm{ng} \mathrm{mL}^{-1}$ ). Typically, standard deviations of validation criteria are lower at high concentrations, where matrix effects are lower. All validation parameters are outlined in ESM Tables S4a-5b. Visualisations of matrix effects in wastewater influent and SPM (via MAE) are displayed in ESM Figure S3. The overlaid chromatograms are scaled by relative intensity, per class, meaning absolute signal suppression can be observed within drug classes. Suppression, and/or poor recovery can be seen for the aminoglycosides (A), some TB drugs (B), carbapenems (C), and two macrolides and their associated metabolites (I).

Recovery from matrix was more varied due to several internal standard compatibilities. Several drugs (INHs, KTCs, BSF, penicillins, DOX, PLFX) suffered from high matrix interference, lowering reproducibility and increasing standard deviations. Some interference was only observed in the channel for product ion 1, or only in solid samples. Consequently, for solid analysis some analytes were re-calibrated using product ion 2 and several were assigned different internal standards (ESM Tables S2 and S6, respectively).

Figures 3 and 4 demonstrate the matrix recoveries for aqueous and solid extractions, respectively; numerical recoveries per matrix are outlined in ESM Table S8. Percentage recoveries were varied throughout both the drug classes and the different matrices. Sulfonamides, macrolides, lincomycins, and quinolones generally performed well, achieving good recoveries from all aqueous extractions. Some exceptions include trimethoprims (TMP and hTMP), which suffered some interference in wastewater samples, and large standard deviations in quinolones (ENR, NOR, PFLX, and OFXo). Sulfonamides, macrolides, and quinolones also achieved the best recoveries from solids, although several metabolites were excluded from the study. Concordance was poor for several analytes, largely attributed to imperfect internal standard pairings. Deuterated or C13 analogues of analytes were used as internal standards. Unfortunately, not all analytes had their labelled equivalents. Therefore, on some occasions, any analyte-specific properties (such as matrix effects, analyte stability, solid-liquid partitioning) affected the analyte and internal standard differently, causing decreased precision and/or accuracy. Percentage recoveries of $<100$ are indicative of insufficient extraction, low analyte stability, or matrix interference. This was observed predominantly in metabolites, $\beta$-lactams, PZA, EMB, 3TC, and more 'dirty' matrices. Poor recoveries may also be biased by less suited internal standards. High standard deviation or a percentage recovery of $>100$ is also indicative of this. If an internal standard has a lower recovery than its paired analyte, the analyte concentration is artificially amplified. Extreme cases of this $(200+\%)$ were excluded, as were outliers, and analytes labelled as only semi-quantitative (e.g. aminoglycosides). Additionally, the method did not recover carbapenems (IPM \& MEM) sufficiently to be quantified in matrix. hPZA and DCS were quantifiable, but the recovered quantities were indistinguishable from matrix blanks - suggesting either chromatographic interference or degradation of the analytical standard.

Method detection limits, calculated from the respective matrix recoveries, were as low as $0.0051 \mathrm{ng} \mathrm{L}^{-1}$ (dmCLI) in aqueous matrices and $0.0024 \mathrm{ng} \mathrm{g}^{-1}$ (ERY) in solids (ESM Table S9). Sulfonamides, macrolides, and azoles achieved the lowest method limits. For example, macrolide MQLs ranged from 0.017 to $10.3 \mathrm{ng} \mathrm{L}^{-1}$ in aqueous and $0.008-20.9 \mathrm{ng} \mathrm{g}^{-1}$ in solids. Several method limits were determined above the analyte's quantitative linear range, 


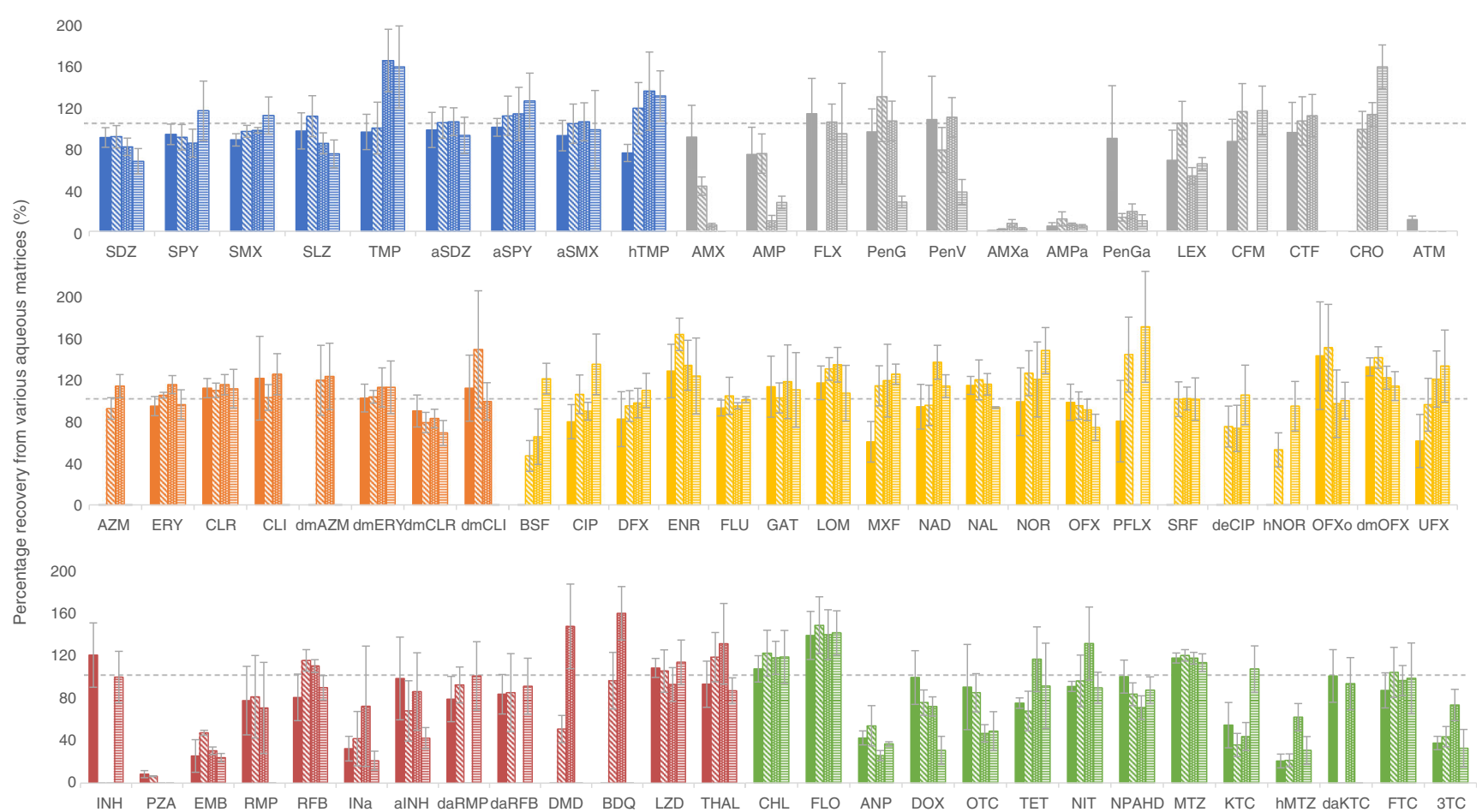

Fig. 3 Method recovery from aqueous matrix, ordered by class (colour) and matrix (bar fill type). The drug classes are sulfonamides (blue); $\beta$ lactams (grey); macrolides and lincomycins (orange); quinolones (yellow); tuberculosis drugs (red); and others: amphenicols, cyclines, nitrofurans, azoles, and antiretrovirals (green). The matrices are Milli-Q water (solid bar); river water (diagonal bar); effluent wastewater (dotted bar); and influent wastewater (horizontal striped bar). Error bars represent replicate standard deviation $(n \leq 18)$.

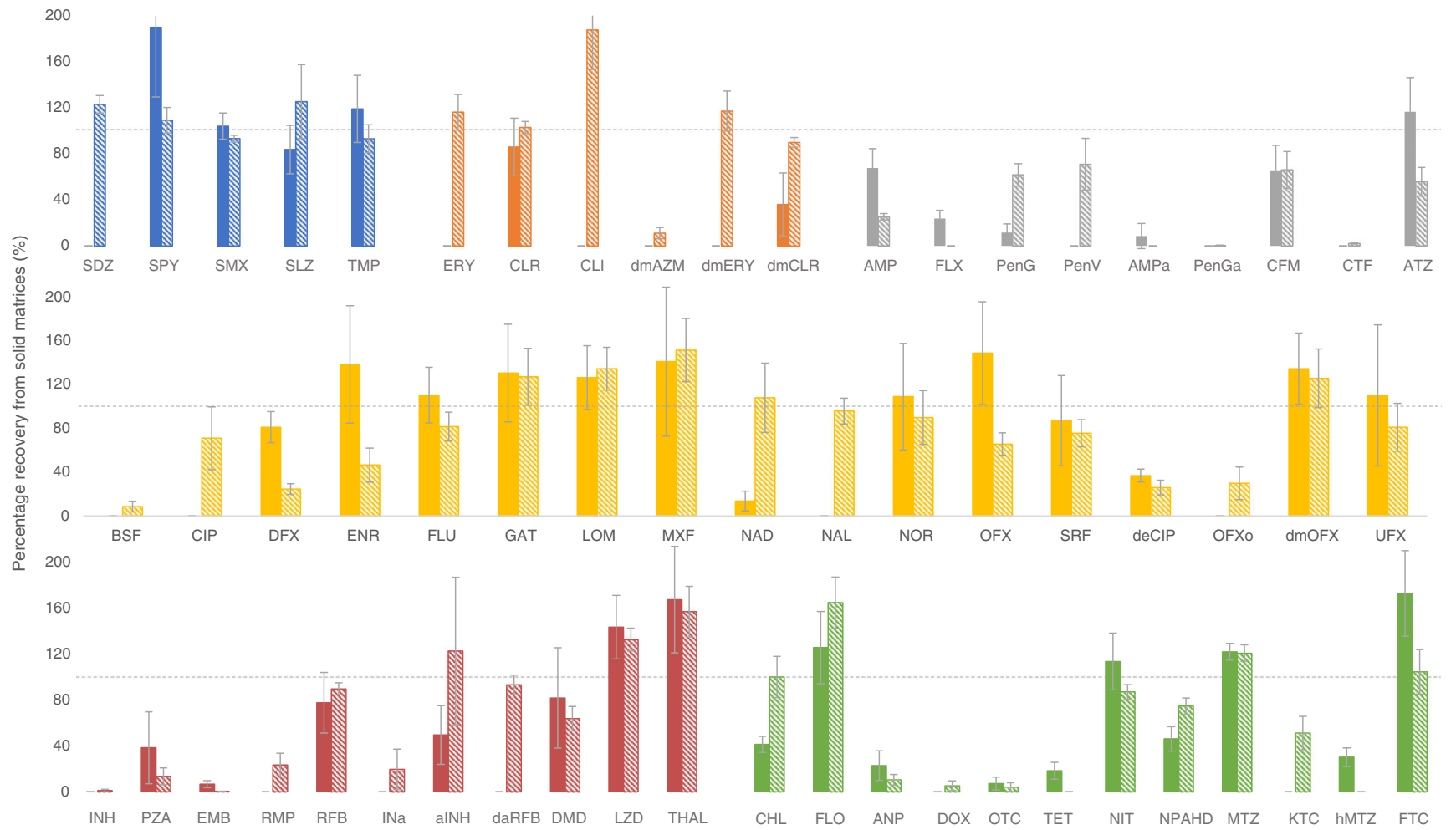

Fig. 4 Method recovery from solid matrix, ordered by class (colour) and matrix (bar fill type). The drug classes are sulfonamides (blue); macrolides and lincomycins (orange); $\beta$-lactams (grey); quinolones (yellow); tuberculosis drugs (red); and others: amphenicols, cyclines,

nitrofurans, azoles, and antiretrovirals (green). The matrices are influent suspended particulate matter (solid bar), and river sediment (diagonal bar). Error bars represent replicate standard deviation $(n \leq 18)$. 


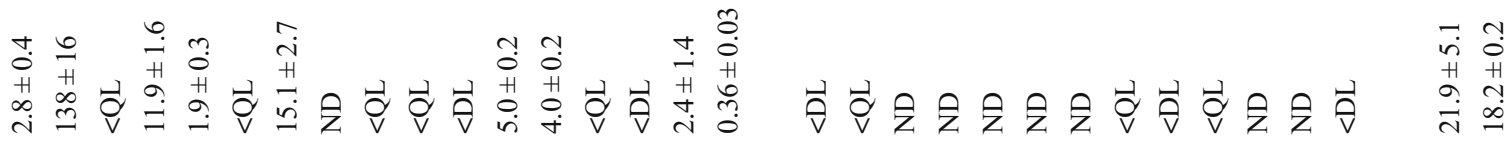

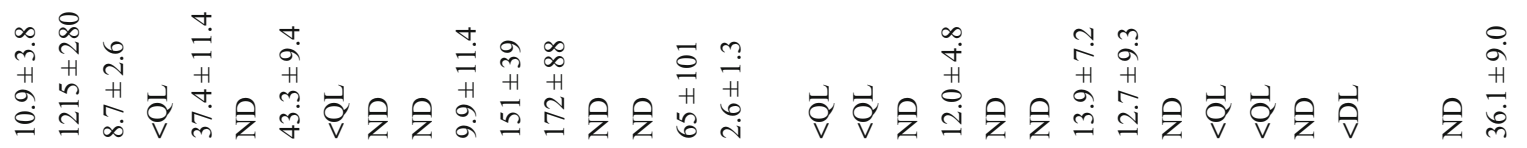

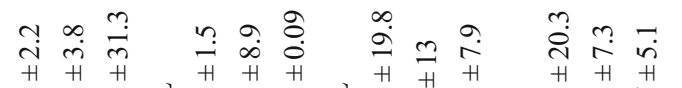

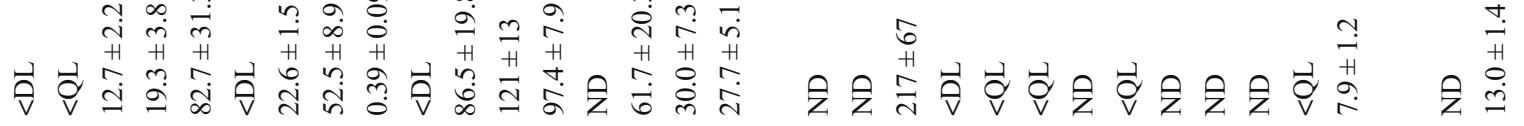

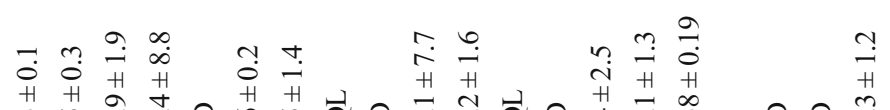

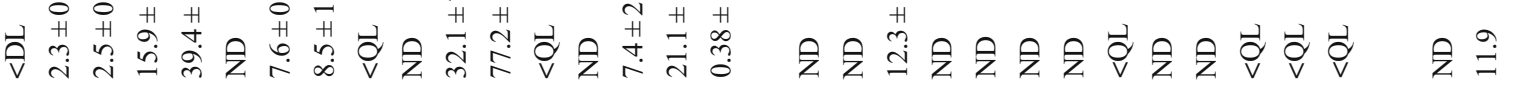

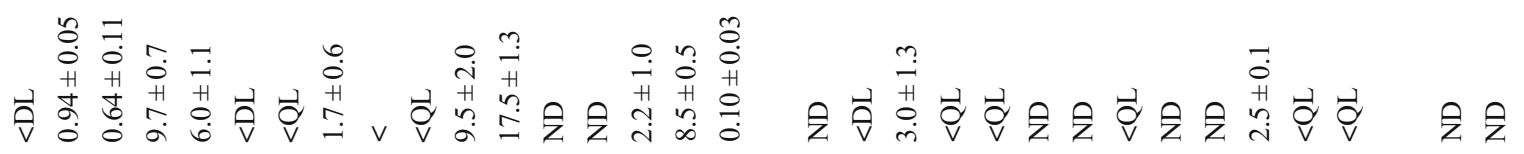

N
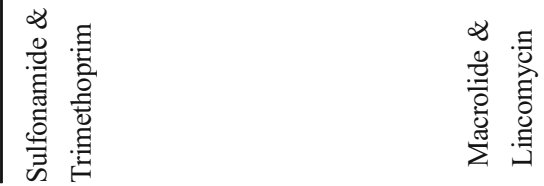

离署

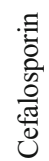

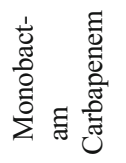









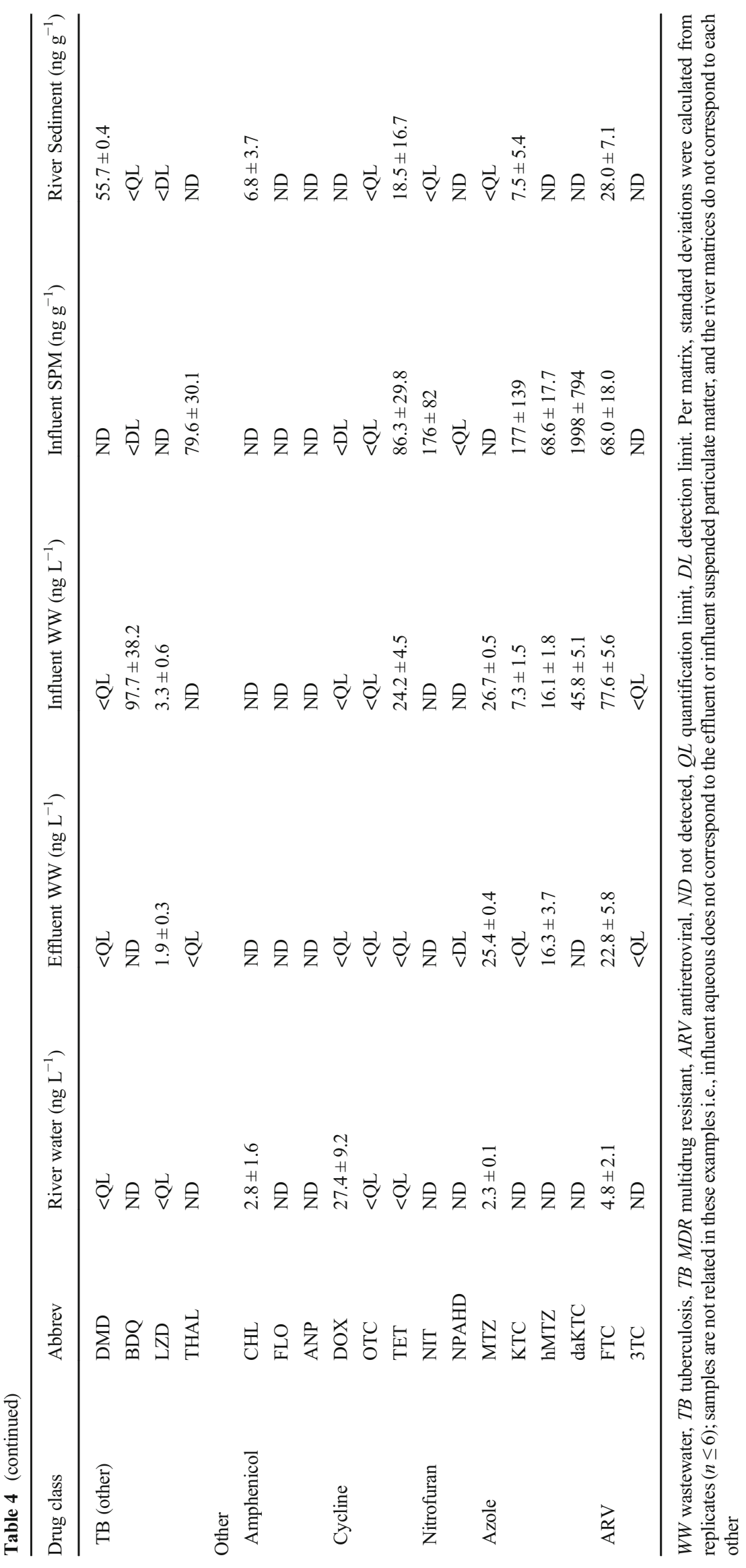


due to very low $(<15 \%)$ recoveries - namely aminoglycosides (TB MDR) and penicillins. Yet overall, the majority of the analytes were considered quantifiable within expected urban sample concentrations.

\section{Application to environmental matrices}

Aqueous matrices included wastewater influent, effluent, and river samples. Solid matrices included wastewater suspended particulate material (SPM), and river sediment. Samples were homogenised and analysed in physical replicate $(n=3)$, whereby each replicate was injected twice. Standard deviations were calculated from the six injections, per matrix. A summary of the sample analyses is displayed in Table 4. Most (60-80\%) antibiotics and their metabolites were found in all studied matrices at concentrations varying from 0.097 to $216 \mathrm{ng} \mathrm{L}^{-1}$ for aqueous, and $0.36-68,911 \mathrm{ng} \mathrm{g}^{-1}$ for solids. Several analytes were found at levels below the corresponding method limits for detection or quantification - these were marked as such rather than quoting numerical values. The results were from singular homogenised samples; therefore, they may not be representative of typical dayto-day concentrations. Further analyses would be required to draw meaningful evaluations of water system health/ contamination.

The main aim of the paper was to develop a method that allows for the simultaneous analysis of several groups of antibiotics in various environmental matrices. Special emphasis was placed on quantification of antibiotic-metabolite pairs. Ability to quantify both antibiotics and their corresponding metabolites in wastewater and in the wider environment can help with understanding patterns of consumption of antibiotics in studied areas as well as providing evidence for direct disposal of antibiotics (e.g. if the antibiotic/metabolite ratio is outside the confidence limit set using human metabolism data). Indeed, antibioticmetabolite pairs were identified and quantified in most environmental matrices. Pairings include SDZ-aSDZ, SPY-aSPY, SMX-aSMX, and TMP-hTMP belonging to sulphonamide/ trimethoprim antibiotic group; AZM-dmAZM, ERY-dmERY, CLR-dmCLR, and CLI-dmCLI in macrolides/lincomycins; AMX-AMXa, AMP-AMPa, and PenG-PenGa in $\beta$-lactams; ENR-CIP-deCIP, NOR-hNOR, OFX-dmOFX/oOFX, and PFLX-UFX in quinolones; INH-aINH/INa, PZA-hPZA, RMPdaRMP, and RFB-daRFB in TB drugs; CHL-ANP in amphenicols; NIT-NPAHD in nitrofurans; and MTZ-hMTZ and KTC-daKTC in azoles. In several cases, metabolite concentrations were higher than the antibiotics themselves.

\section{Conclusions}

This method was developed for the quantification of 58 parent drugs (54 antibiotics, 1 antifungal, 2 antiretrovirals, and 1 TB-relevant drug) and 26 metabolites (25 antibiotics and 1 antifungal). Drug compositions are often comprised of several chemical forms, for example capreomycins IA and IB. Drugs with significant proportions $(>5 \%)$ of different mass forms were designed and validated separately, but the results were combined and quoted for the encompassing drug. Quantitative analysis was achieved for the majority of targets for the aqueous $(66 ;>78 \%)$ and solid $(58 ;>69 \%)$ calibrations but was regarded as only semi-quantitative for the remaining targets. High sample throughput was a requirement of this LC-MS method due to research design involving analysis of a large number of samples in a high-demand research laboratory. The chromatography was compressed and optimised into a 19-min gradient, for spectrometric analysis of 116 MRM channels.

Recovery of analytes from different matrices was variable. The number of analytes successfully recovered was determined per matrix. From 'clean' water (Milli-Q or river water), 64 were recovered quantitatively; due to validation and recovery performance, 18 were considered semi-quantitative, and 2 were not detectable. For wastewater (influent or effluent), the numbers of analytes were 63,19, and 2, respectively, and for solids (SPM or sediment), they were 45,37 , and 2 , respectively. Recovery tests were performed using one homogenised sample in triplicate per matrix. In these samples, several analytes were quoted as above their linear quantitative ranges. If the baseline level of analyte was lower, or a dilution was made, those analytes would likely produce quantitative results. Based on matrix-specific recoveries, MQLs were determined as low as $0.017 \mathrm{ng} \mathrm{L}^{-1}$ in river water, $0.044 \mathrm{ng} \mathrm{L}^{-1}$ in wastewater, $0.008 \mathrm{ng} \mathrm{g}^{-1}$ in river sediment, and $0.009 \mathrm{ng} \mathrm{g}^{-1}$ in wastewater SPM. Overall, these results demonstrate the success and versatility of the method in terms of the high number of analytes and quantitation of different environmental matrices.

In environmental samples, most analytes (60-80\%) were quantified in all studied matrices at concentrations varying from 0.097 to $216 \mathrm{ng} \mathrm{L}^{-1}$ for aqueous and $0.36-68,911 \mathrm{ng} \mathrm{g}^{-1}$ for solids. Another feature of this method, and a distinction from a lot of existing WBE research, was the inclusion of drug metabolites. Several antibiotic-metabolite pairs were successfully quantified. These include four pairings belonging to sulphonamide/trimethoprim antibiotic group; four in macrolides/lincomycins; three in $\beta$-lactams; four in quinolones; four in TB drugs; one in amphenicols; one in nitrofurans; and two in azoles. The ability to quantify both antibiotics and their corresponding metabolites in wastewater and in the wider environment is of particular importance; it can help with understanding patterns of consumption of antibiotics in studied areas, as well as providing evidence for direct disposal of antibiotics. Understanding AB-MET relationships during wastewater treatment and in the wider environment will help with unravelling complexities of the environmental fate of antibiotics and resulting risks they pose, both in the context of chemical toxicity and AMR. 
Supplementary Information The online version contains supplementary material available at https://doi.org/10.1007/s00216-021-03573-4.

Funding Elizabeth Holton received funding for her $\mathrm{PhD}$ from the University of Bath. The support of Engineering and Physical Sciences Research Council (EP/P028403/1) is greatly appreciated.

Data Availability The datasets generated during and/or analysed during the current study are available from the corresponding author on reasonable request.

Code availability Not applicable

\section{Declarations}

Competing interests The authors declare no competing interests.

Open Access This article is licensed under a Creative Commons Attribution 4.0 International License, which permits use, sharing, adaptation, distribution and reproduction in any medium or format, as long as you give appropriate credit to the original author(s) and the source, provide a link to the Creative Commons licence, and indicate if changes were made. The images or other third party material in this article are included in the article's Creative Commons licence, unless indicated otherwise in a credit line to the material. If material is not included in the article's Creative Commons licence and your intended use is not permitted by statutory regulation or exceeds the permitted use, you will need to obtain permission directly from the copyright holder. To view a copy of this licence, visit http://creativecommons.org/licenses/by/4.0/.

\section{References}

1. Gonzalez-Marino I, Baz-Lomba JA, Alygizakis NA, Andres-Costa MJ, Bade R, Bannwarth A, et al. Spatio-temporal assessment of illicit drug use at large scale: evidence from 7 years of international wastewater monitoring. Addiction. 2020;115(1):109-20.

2. Ort C, van Nuijs ALN, Berset J-D, Bijlsma L, Castiglioni S, Covaci A, et al. Spatial differences and temporal changes in illicit drug use in Europe quantified by wastewater analysis. Addiction. 2014;109(8):1338-52.

3. Thomas KV, Bijlsma L, Castiglioni S, Covaci A, Emke E, Grabic $\mathrm{R}$, et al. Comparing illicit drug use in 19 European cities through sewage analysis. Science of the Total Environment. 2012;432:432 9.

4. Rice J, Kannan AM, Castrignano E, Jagadeesan K, KasprzykHordern B. Wastewater-based epidemiology combined with local prescription analysis as a tool for temporal monitoring of drugs trends-A UK perspective. Science of the Total Environment. $2020 ; 735$.

5. Rousis NI, Gracia-Lor E, Zuccato E, Bade R, Baz-Lomba JA, Castrignano E, et al. Wastewater-based epidemiology to assess pan-European pesticide exposure. Water Research. 2017;121: $270-9$.

6. Rousis NI, Zuccato E, Castiglioni S. Wastewater-based epidemiology to assess human exposure to pyrethroid pesticides. Environment International. 2017;99:213-20.

7. Lopardo L, Petrie B, Proctor K, Youdan J, Barden R, KasprzykHordern B. Estimation of community-wide exposure to bisphenol A via water fingerprinting. Environment International. 2019;125:18 .
8. Kasprzyk-Hordern B, Proctor K, Jagadeesan K, Lopardo L, O’Daly KJ, Standerwick R, et al. Estimation of community-wide multichemical exposure via water-based chemical mining: key research gaps drawn from a comprehensive multi-biomarker multi-city dataset. Environment International. 2021;147:106331.

9. Been F, Bastiaensen M, Lai FY, Libousi K, Thomaidis NS, Benaglia $\mathrm{L}$, et al. Mining the chemical information on urban wastewater: monitoring human exposure to phosphorus flame retardants and plasticizers. Environ Sci Technol. 2018;52(12):6996-7005.

10. Gracia-Lor E, Zuccato E, Hernandez F, Castiglioni S. Wastewaterbased epidemiology for tracking human exposure to mycotoxins. J Hazard Mater. 2020;382:121108.

11. Ahmed W, Tscharke B, Bertsch PM, Bibby K, Bivins A, Choi P, et al. SARS-CoV-2 RNA monitoring in wastewater as a potential early warning system for COVID-19 transmission in the community: a temporal case study. Sci Total Environ. 2021;761:144216.

12. Bivins A, North D, Ahmad A, Ahmed W, Alm E, Been F, et al. Wastewater-based epidemiology: global collaborative to maximize contributions in the fight against COVID-19. Environmental Science \& Technology. 2020;54(13):7754-7.

13. Castrignano E, Yang ZE, Feil EJ, Bade R, Castiglioni S, Causanilles A, et al. Enantiomeric profiling of quinolones and quinolones resistance gene qnrS in European wastewaters. Water Research. 2020;175:115653.

14. Castrignano E, Kannan AM, Proctor K, Petrie B, Hodgen S, Feil $\mathrm{EJ}$, et al. (Fluoro)quinolones and quinolone resistance genes in the aquatic environment: a river catchment perspective. Water Research. 2020;182:116015.

15. Munita JM, Arias CA. Mechanisms of antibiotic resistance. Microbiol Spectr. 2016;4(2):10.1128/microbiolspec.VMBF-00162015.

16. Bouki C, Venieri D, Diamadopoulos E. Detection and fate of antibiotic resistant bacteria in wastewater treatment plants: a review. Ecotox Environ Safe. 2013;91:1-9.

17. Wales AD, Davies RH. Co-selection of resistance to antibiotics, biocides and heavy metals, and its relevance to foodborne pathogens. Antibiotics-Basel. 2015;4(4):567-604.

18. Castrignanò E, Kannan AM, Feil EJ, Kasprzyk-Hordern B. Enantioselective fractionation of fluoroquinolones in the aqueous environment using chiral liquid chromatography coupled with tandem mass spectrometry. Chemosphere. 2018;206:376-86.

19. Riva F, Zuccato E, Pacciani C, Andrea C, Castiglioni S. A multiresidue analytical method for extraction and analysis of pharmaceuticals and other selected emerging contaminants in sewage sludge. Analytical Methods. 2021;13(4):526-35.

20. Bajkacz S, Felis E, Kycia-Slocka E, Harnisz M, Korzeniewska E. Development of a new SLE-SPE-HPLC-MS/MS method for the determination of selected antibiotics and their transformation products in anthropogenically altered solid environmental matrices. Sci Total Environ. 2020;726:138071.

21. Wang CF, Li XW, Lv ZQ, Wang YY, Ke YB, Xia X. Determination of carbapenems in water samples by UHPLC-MS/ MS. Journal of Separation Science. 2020;43(12):2321-9.

22. Siedlewicz G, Borecka M, Bialk-Bielinska A, Sikora K, Stepnowski P, Pazdro K. Determination of antibiotic residues in southern Baltic Sea sediments using tandem solid-phase extraction and liquid chromatography coupled with tandem mass spectrometry. Oceanologia. 2016;58(3):221-34.

23. Solliec M, Roy-Lachapelle A, Sauve S. Development of a suspect and non-target screening approach to detect veterinary antibiotic residues in a complex biological matrix using liquid chromatography/high-resolution mass spectrometry. Rapid Communications in Mass Spectrometry. 2015;29(24):2361-73.

24. Xue M, Wu HC, Liu SY, Huang XH, Jin Q, Ren R. Simultaneous determination of 44 pharmaceutically active compounds in water samples using solid-phase extraction coupled with ultra- 
performance liquid chromatography-tandem mass spectrometry. Analytical and Bioanalytical Chemistry. 2020;412(1):203-22.

25. Kim C, Ryu HD, Chung EG, Kim Y. Determination of 18 veterinary antibiotics in environmental water using high-performance liquid chromatography-q-orbitrap combined with on-line solidphase extraction. Journal of Chromatography B-Analytical Technologies in the Biomedical and Life Sciences. 2018;1084: 158-65.

26. Axel M, Ewelina K, Jenny-Maria B, Leif K. An online SPE LCMS/MS method for the analysis of antibiotics in environmental water. Environmental Science and Pollution Research. 2017;24(9):8692-9.

27. Sharma D, Dhuriya YK, Deo N, Bisht D. Repurposing and revival of the drugs: a new approach to combat the drug resistant tuberculosis. Front Microbiol. 2017;8:2452.
28. Keddie S, Bharambe V, Jayakumar A, Shah A, Sanchez V, Adams A, et al. Clinical perspectives into the use of thalidomide for central nervous system tuberculosis. Eur J Neurol. 2018;25(11):1345-51.

29. Petrie B, Youdan J, Barden R, Kasprzyk-Hordern B. Multi-residue analysis of 90 emerging contaminants in liquid and solid environmental matrices by ultra-high-performance liquid chromatography tandem mass spectrometry. J Chromatogr A. 2016;1431:64-78.

30. FDA. Bioanalytical method validation: guidance for industry. 2018 May 2018 (https://www.fda.gov/files/drugs/published/ Bioanalytical-Method-Validation-Guidance-for-Industry.pdf).

Publisher's note Springer Nature remains neutral with regard to jurisdictional claims in published maps and institutional affiliations. 